\title{
Mechanism of destruction of transport barriers in geophysical jets with Rossby waves
}

\author{
M.Yu. Uleysky, M.V. Budyansky, and S.V. Prants \\ Pacific Oceanological Institute \\ of the Russian Academy of Sciences, \\ 43 Baltiiskaya st., 690041 Vladivostok, Russia
}

\begin{abstract}
The mechanism of destruction of a central transport barrier in a dynamical model of a geophysical zonal jet current in the ocean or the atmosphere with two propagating Rossby waves is studied. We develop a method for computing a central invariant curve which is an indicator of existence of the barrier. Breakdown of this curve under a variation of the Rossby wave amplitudes and onset of chaotic cross-jet transport happen due to specific resonances producing stochastic layers in the central jet. The main result is that there are resonances breaking the transport barrier at unexpectedly small values of the amplitudes that may have serious impact on mixing and transport in the ocean and the atmosphere. The effect can be found in laboratory experiments with azimuthal jets and Rossby waves in rotating tanks under specific values of the wave numbers that are predicted in the theory.

PACS numbers: 05.45.-a,05.60.Cd,47.52.+j
\end{abstract}

Transport and mixing of water (air) masses and their characteristics play a crucial role in the ocean and atmosphere dynamics. In the Lagrangian approach a particle with the position $\vec{r}$ is advected by an Eulerian velocity field $\vec{v}(\vec{r}, t)$

$$
\frac{d \vec{r}}{d t}=\vec{v}(\vec{r}, t)
$$

It is known that a simple deterministic velocity field may produce practically unpredictable particle trajectories, the phenomenon known as chaotic advection [1 3 ].

We study theoretically and numerically horizontal cross-jet transport in geophysical zonal flows. To list a few we mention the Gulf Stream in the Atlantic, the Kuroshio in the Pacific, and the polar night Antarctic jet in the atmosphere, which are the jet currents separating water (air) masses with different physical properties. Transport of particles across a geophysical jet is of crucial importance and may cause, for example, depletion of ozone in the atmosphere and heating and freshing of waters in the ocean. The velocity fields of real flows are not, of course, regular, but if the Eulerian correlation time is large as compared to the Lagrangian one, the problem may be treated in the framework of chaotic advection concept.

The equations of motion of a passive particle with coordinates $x$ and $y$ advected by a two-dimensional incompressible flow with a stream function $\Psi$ are known to have a Hamiltonian form [1]

$$
\frac{d x}{d t}=u(x, y, t)=-\frac{\partial \Psi}{\partial y}, \quad \frac{d y}{d t}=v(x, y, t)=\frac{\partial \Psi}{\partial x},
$$

with the phase space being the position space for advected particles. Chaotic mixing and transport in jet flows have been extensively studied with kinematic models, where the velocity field is a given function of $x, y$ and $t$ imitating real flows (see [3 $[6]$ and references therein), and with dynamical models conserving the potential vorticity (see [3, 7- 9] and references therein). The problem has been studied as well in laboratory where azimuthal jets with Rossby waves have been produced in rotating tanks [10, 11]. It has been found both numerically and experimentally that fluid is effectively mixed along the jet, but in common opinion a large gradient of the potential vorticity in the central part prevents transport across the jet. A technique, based on computing the finite-scale Lyapunov exponent, has been found useful in Ref. 12] to detect the presence of cross-jet barriers in kinematic models. A comparison of properties of cross-jet transport in kinematic and dynamical models of atmospheric zonal jets has been done recently in Ref. 13]. Up to now, the transport barrier has been shown numerically [5, 9] to be broken only with so large values of the wave amplitudes that are beyond of the validity of linear models and can be hardly observed in real flows.

The aim of the paper is to prove that cross-jet transport under appropriate conditions is possible at comparatively small values of the wave amplitudes and, therefore, may occur in geophysical jets. We develop a general method to detect a core of the transport barrier and find a mechanism of its destruction using the dynamical model of a zonal jet flow with two propagating Rossby waves. The method comprises the identification of a central invariant curve (CIC), which is an indicator of existence of the barrier, finding certain resonant conditions for its destruction at given values of the wave numbers, and detection of cross-jet transport.

Motion of two-dimensional incompressible fluid in the rotating frame is governed by the equation for conserving potential vorticity $(\partial / \partial t+\vec{v} \cdot \vec{\nabla}) \Pi=0$. In the quasigeostrophic approximation [14], one gets $\Pi=\nabla^{2} \Psi+\beta y$, where $\beta$ is the Coriolis parameter. The $x$ axis is chosen along the zonal flow, from the west to the east and $y$ - along the gradient from the south to the north. Barotropic perturbations of zonal flows produce Rossby waves which have an essential impact on transport and mixing in the ocean and the atmosphere [14]. The stream 
function is sought in the form

$$
\Psi=\Psi_{0}+\Psi_{\text {int }}=\Psi_{0}(y)+\sum_{j} \Phi_{j}(y) e^{i k_{j}\left(x-c_{j} t\right)},
$$

where $\Psi_{0}$ describes a zonal flow and $\Psi_{\text {int }}$ is its perturbation which is supposed to be a superposition of zonal running Rossby waves. After substituting (3) in the equation for the potential vorticity and a linearization, one gets the Rayleigh-Kuo equation [15]

$$
\left(u_{0}-c_{j}\right)\left(\frac{d^{2} \Phi_{j}}{d y^{2}}-k_{j}^{2} \Phi_{j}\right)+\left(\beta-\frac{d^{2} u_{0}}{d y^{2}}\right) \Phi_{j}=0
$$

where the zonal velocity $u_{0}=-d \Psi_{0} / d y$ has a single extremum at $y=0$. If one takes the following zonal-velocity profile (the Bickley jet [8]):

$$
u_{0}(y)=U_{0} \operatorname{sech}^{2} \frac{y}{D},
$$

then Eq. (4) admits two neutrally stable solutions

$$
\Phi_{j}(y)=A_{j} U_{0} D \operatorname{sech}^{2} \frac{y}{D}, \quad j=1,2,
$$

where $U_{0}$ is the maximal velocity in the flow, $D$ is a measure of its width, and $A_{j}$ are the wave amplitudes. It is easy to check that (5) and (6) are compatible with (4) if there is the following condition for the phase velocities:

$$
c_{1,2}=\frac{U_{0}}{3}(1 \pm \alpha), \quad \alpha \equiv \sqrt{1-\beta^{*}}, \quad \beta^{*} \equiv \frac{3 D^{2} \beta}{2 U_{0}},
$$

which are connected with the wave numbers by the dispersion relation $c_{1,2}=U_{0} D^{2} k_{1,2}^{2} / 6$. Two neutrally stable Rossby waves exist if $\beta D^{2} / U_{0}<2 / 3$.

Thus, the stream function of the zonal flow with two Rossby waves, satisfying the conservation of the potential vorticity, has the form

$$
\begin{aligned}
& \Psi(x, y, t)=-U_{0} D\left(\tanh \frac{y}{D}-\operatorname{sech}^{2} \frac{y}{D} \times\right. \\
& \left.\times\left[A_{1} \cos k_{1}\left(x-c_{1} t\right)+A_{2} \cos k_{2}\left(x-c_{2} t\right)\right]\right) .
\end{aligned}
$$

One of the task of this paper is to present results in the form allowing a comparison with laboratory experiments [10, 11] in which an azimuthal jet at the radius $R$ with Rossby waves with the wave numbers $n_{1}$ and $n_{2}$ has been produced:

$$
k_{1,2}=\frac{n_{1,2}}{R}, \quad c_{1,2}=\frac{U_{0} D^{2}}{6 R^{2}} n_{1,2}^{2} .
$$

Let it be $n_{1}>n_{2}$, and the wave with $n_{1}$ is called the first one. Let the wave numbers be represented as $n_{1}=m N_{1}$ and $n_{2}=m N_{2}$, where $m \neq 1$ is the greatest common divisor and $N_{1} / N_{2}$ is an irreducible fraction. Introducing new coordinates $x^{\prime}, y^{\prime}$, and $t^{\prime}$

$$
x=\frac{\left(x^{\prime}+C_{2} t^{\prime}\right) R}{m}, \quad y=D y^{\prime}, \quad t=\frac{R}{m U_{0}} t^{\prime},
$$

we rewrite the stream function (8) in the frame moving with the phase velocity of the first wave

$$
\begin{array}{r}
\Psi^{\prime}\left(x^{\prime}, y^{\prime}, t^{\prime}\right)=-\tanh y^{\prime}+A_{1} \operatorname{sech}^{2} y^{\prime} \cos \left(N_{1} x^{\prime}\right)+ \\
+A_{2} \operatorname{sech}^{2} y^{\prime} \cos \left(N_{2} x^{\prime}+\omega_{2} t^{\prime}\right)+C_{2} y^{\prime}
\end{array}
$$

where

$$
\omega_{2} \equiv \frac{2 N_{2}\left(N_{1}^{2}-N_{2}^{2}\right)}{3\left(N_{1}^{2}+N_{2}^{2}\right)}, \quad C_{2} \equiv \frac{2 N_{1}^{2}}{3\left(N_{1}^{2}+N_{2}^{2}\right)} .
$$

Thus, we get the stream function (11) with the control parameters $N_{1}$ and $N_{2}$ which are specified by the four experimental parameters: $U_{0}, \beta, D$, and $R$. One can now study cross-jet transport with any combination of the wave numbers $n_{1}$ and $n_{2}$ that can be realized in a laboratory experiment by adjusting the radius $R$, the jet width $D$, the maximal velocity $U_{0}$, and the Coriolis-like parameter $\beta[10,11]$.

The advection equations (2) with the stream function (11) have the form

$$
\begin{gathered}
\frac{d x}{d t}=-C_{2}+\operatorname{sech}^{2} y\left[1+2 A_{1} \tanh y \cos \left(N_{1} x\right)+\right. \\
\left.+2 A_{2} \tanh y \cos \left(N_{2} x+\omega_{2} t\right)\right], \\
\frac{d y}{d t}=-\operatorname{sech}^{2} y\left[A_{1} N_{1} \sin \left(N_{1} x\right)+A_{2} N_{2} \sin \left(N_{2} x+\omega_{2} t\right)\right],
\end{gathered}
$$

where we omitted the primes over $x, y$, and $t$. If the amplitude of the second wave is zero, $A_{2}=0$, then the set (13) is integrable. The phase portrait of the steady flow with $A_{1}=0.2416, N_{1}=5$ and $N_{2}=1$ is shown in Fig. 1 in the frame moving with the phase velocity of the first wave. The eastward jet is situated between two chains with five vortices. The southern and northern peripheral currents are westward in the moving frame. In a steady flow all the particles follow streamlines. At $A_{2}>0$, chaos may arise in a typical way: a stochastic layer appears at the place of the broken separatrices (Fig. 1 b and c).

At odd values of $N_{1}$ and $N_{2}$, Eqs. (13) have the two symmetries

$$
\hat{S}:\left\{\begin{array}{l}
\tilde{x}=\pi+x, \\
\tilde{y}=-y,
\end{array} \quad \hat{I}_{0}:\left\{\begin{array}{l}
\tilde{x}=-x, \\
\tilde{y}=y,
\end{array}\right.\right.
$$

which are involutions, i. e., $\hat{S}^{2}=1$ and $\hat{I}_{0}^{2}=1$. Solving the equation $\hat{I}_{0}\left(x_{j}, y_{j}\right)=\hat{S}\left(x_{j}, y_{j}\right), j=1,2$, one gets indicator points [16]: $\left(x_{1}=\pi / 2, y_{1}=0\right)$ and $\left(x_{2}=3 \pi / 2\right.$, $\left.y_{2}=0\right)$. Iterating them, we construct a CIC [17] in the central part of the jet which is the last transport barrier in the sense that the CIC breaks down and is replaced by a stochastic layer with variation of the wave amplitudes. We illustrate this in Fig. 1. At $A_{2}=0.09$, the CIC together with neighboring invariant curves forms a narrow transport barrier (Fig. 10). We define a CIC as a curve which is invariant under the operator $\hat{S}$ and the evolution operator over the period $2 \pi / \omega_{2}$. The CIC separates the northern and southern parts of the flow. At 

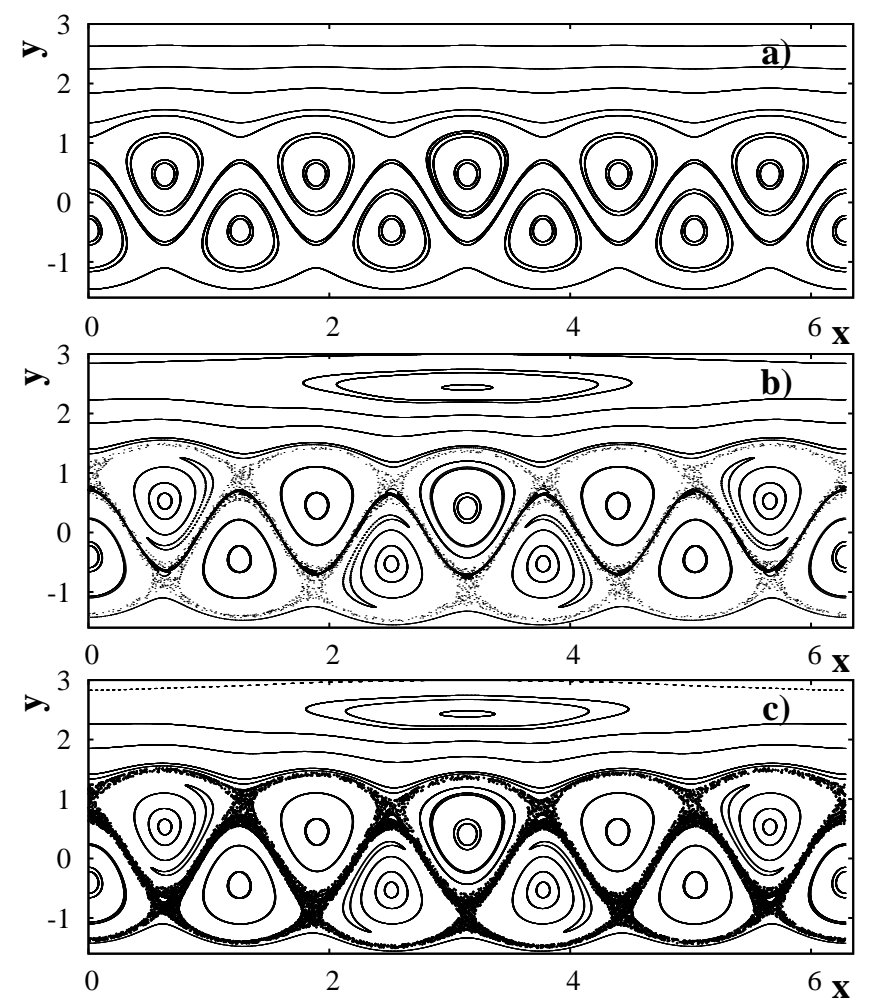

FIG. 1. (a) $A_{2}=0$. Phase portrait of the steady jet flow with $N_{1}=5$ and $N_{2}=1$ in the moving frame. (b) $A_{2}=0.09$. CIC (the bold curve) is a barrier to transport across the jet. (c) $A_{2}=0.095$. Destruction of CIC and onset of cross-jet transport.

$A_{2}=0.095$, the CIC breaks down, and cross-jet transport becomes possible (Fig. 11).

It is reasonable to suppose that destruction of CIC is caused by a ballistic resonance between the maximal frequency of the particle motion in the central jet and the perturbation frequency $\omega_{2}$. The first one is estimated from Eq. (13) to be $f_{1} \simeq-C_{2}+1$, and the second one is given by (12). So, the approximate condition of the ballistic resonance is

$$
\frac{f_{1}}{\omega_{2}}=\frac{N_{1}^{2}+3 N_{2}^{2}}{2 N_{2}\left(N_{1}^{2}-N_{2}^{2}\right)} .
$$

At small amplitudes, this ratio gives an approximate estimate for the CIC winding number $w$ [17]. Equating the right-hand side of Eq. (15) to a rational number, one finds those values of the wave numbers $N_{1}$ and $N_{2}$ for which the CIC is strongly influenced by the corresponding resonance, and, therefore, cross-jet transport becomes possible.

In order to reveal a scenario for CIC destruction we plot in Fig. 2 the dependencies of $w$ and the maximal deviation of iterations of the indicator point along the y-axis, $\left|y_{\max }\right|$, on $A_{1}$ and $A_{2}$ for the pair $\left(N_{1}=5, N_{2}=\right.$ 1 ). The bifurcation curves with the winding numbers corresponding to certain resonances are shown in Fig. 2 h.
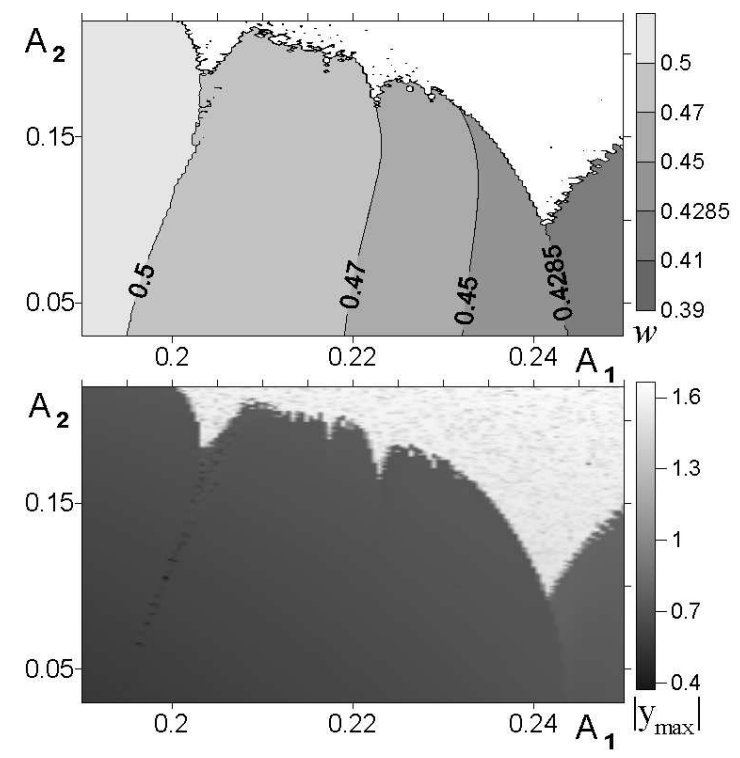

FIG. 2. Diagrams of (a) the winding number $w$ and (b) the maximal deviation of iterations of the indicator point along the y-axis, $\left|y_{\max }\right|$, in the space of the Rossby wave amplitudes $A_{1}$ and $A_{2}$. White zone: regime with cross-jet transport.

The even $2: 1(w=0.5)$ and odd $7: 3(w=0.4285)$ ones produce two deep and wide spikes in the plots. White color codes those values of the amplitudes $A_{1,2}$ at which a $\mathrm{CIC}$ is broken. Comparing Figs. $2 \mathrm{a}$ and $\mathrm{b}$, we see that the zone with broken CIC in Fig. 2a correspond to the values $\left|y_{\max }\right| \simeq 1.5$ in Fig. 2 $b$, i.e., iterations of points, situated initially in the jet core, cover the region of the size of order $\simeq 3$ jet's half-width. It means breakdown of central transport barrier at those values of $A_{1,2}$ at which the CIC is broken. Figure 2 demonstrates clearly that destruction of the transport barrier may happen at comparatively small values of the wave amplitudes $\left(A_{1,2}<1\right)$. Our model is essentially a linear one, and the Rayleigh-Kuo equation is valid to first order in the wave amplitudes.

To illustrate the mechanism of destruction of CIC we study the topology of the phase space near the islands of the resonance $7: 3$ (see the spike with $w=0.4285 \ldots$ in Fig. (2). Let us fix $A_{1}=0.2418$ and gradually increase $A_{2}$ away from zero. In the range $0<A_{2}<0.088$ the smooth CIC and neighboring invariant curves form a transport barrier (Fig. [3a). At $A_{2} \simeq 0.088$, invariant manifolds of hyperbolic orbits of the resonance $7: 3$ cross each other, the CIC breaks down, and there appears at its place a narrow stochastic layer locked between remained invariant curves (Fig. 3b). When $A_{2}$ increases further islands of the resonance $7: 3$ diverge, and a meandering CIC appears again between them (see Fig. 3. at $A_{2}=0.09$ ). At $A_{2}>0.095$, CIC and surrounding invariant curves are destroyed, and cross-jet transport becomes possible in a wide range of the $y$ coordinate (Fig. 3). Thus, existence of a CIC is a sufficient but not necessary condition for existence of a transport barrier. Animation of metamorphoses of topology of the transport barrier and its de- 
struction at a fixed value of one of the wave amplitudes and variation of the other one can be found in Ref. [18].
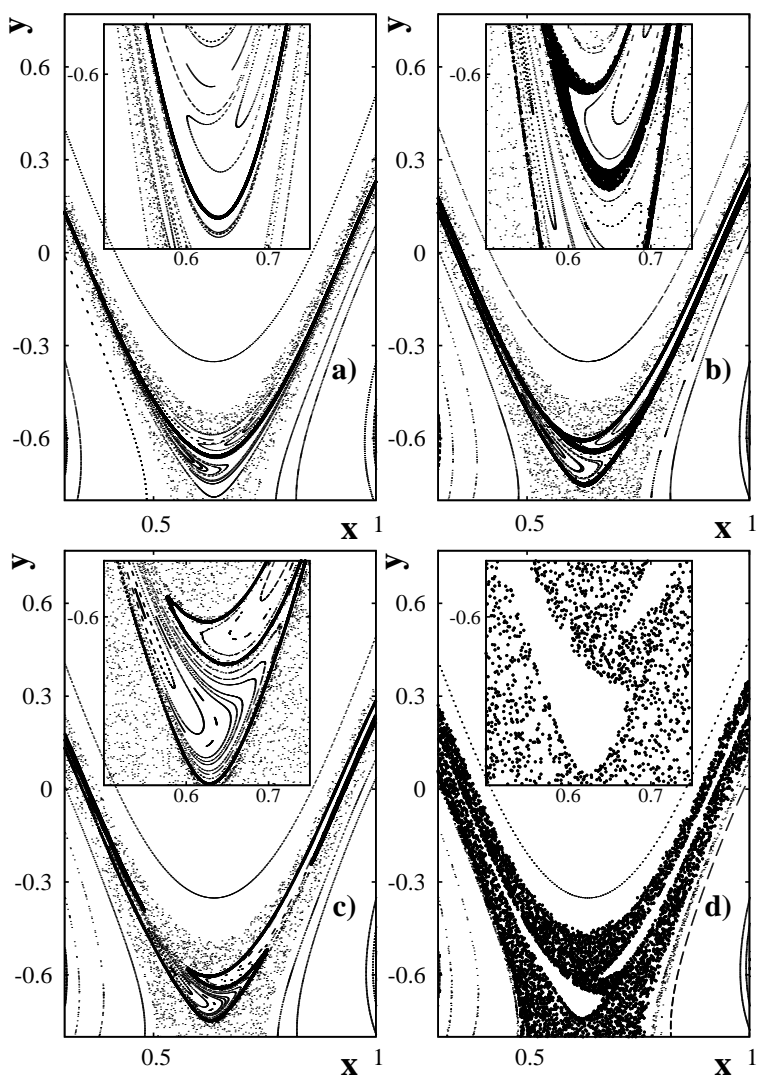

FIG. 3. Mechanism of CIC destruction and onset of cross-jet transport. (a) $A_{2}=0.087$. Smooth CIC and neighboring invariant curves form a transport barrier. (b) $A_{2}=0.088$. A narrow stochastic layer (shadowed strip) appears at the place of the broken CIC. (c) $A_{2}=0.09$. CIC appears again as a meandering curve. (d) $A_{2}=0.1$. Breakdown of CIC and onset of cross-jet transport. Insets show magnification of the phase space region nearby the resonance $7: 3$.
In conclusion we discuss briefly a possibility for checking main results of our work in laboratory experiments on chaotic advection in rotating fluid [10, 11] imitating nonlinear geostrophical geophysical flows in the ocean and the atmosphere. An azimuthal jet with Rossby waves was produced by the action of the Coriolis force on radially pumped fluid in a rotating tank with a slope imitating the $\beta$-effect on the rotating Earth. The measured velocity field was rather well approximated by the model stream function (8) [11]. Rapid mixing on either side of the jet was observed for a quasiperiodic flow, but no significant transport was observed across the jet. In our opinion the reason is that the experiments have been carried out under conditions that are far away from the resonances which are capable of destroying the central transport barrier at the values of the Rossby wave numbers realized in the experiment. The results obtained in this paper allow to specify those values of the control parameters of the flow, the Rossby wave numbers, for which there exist specific resonances capable of destroying transport barriers at comparatively small values of the wave amplitudes. Our recommendation to observe cross-jet transport in laboratory is to produce an azimuthal jet and Rossby waves with odd wave numbers whose values differ significantly from each other, say $\left(N_{1}=5, N_{2}=1\right)$ or $\left(N_{1}=7, N_{2}=3\right)$.

The work was supported partially by the Program "Fundamental Problems of Nonlinear Dynamics" of the Russian Academy of Sciences and by the Russian Foundation for Basic Research (project no. 09-05-98520).
[1] H. Aref, J. Fluid Mech. 143, 1 (1984).

[2] J. M. Ottino, Annu. Rev. Fluid Mech. 22, 207 (1990).

[3] K. V. Koshel and S. V. Prants, Physics-Uspekhi 49, 1151 (2006). K. V. Koshel and S. V. Prants, Chaotic advection in the ocean (ICS, Moscow-Izhevsk, 2008) [in Russian].

[4] R. M. Samelson, J. Phys. Oceanogr. 22, 431 (1992).

[5] F. Raynal and S. Wiggins, Physica D 223, 7 (2006).

[6] M. Yu. Uleysky, M. V. Budyansky, and S. V. Prants, Chaos 17, 024703 (2007).

[7] R. T. Pierrehumbert, Geophys. Astrophys. Fluid Dyn. 58, 285 (1991).

[8] D. Del-Castillo-Negrete and P. J. Morrison, Phys. Fluids A 5, 948 (1993).

[9] I. I. Rypina, M. G. Brown, F. J. Beron-Vera, H. Kozak, M. J. Olascoaga, and I. A. Udovydchenkov, J. Atmos. Sci. 64, 3595 (2007).

[10] J. Sommeria, S. D. Meyers, and H. L. Swinney, Nature
337, 58 (1989).

[11] T. H. Solomon, W. J. Holloway, and H. L. Swinney, Phys. Fluids A 5, 1971 (1993).

[12] G. Boffetta, G. Lacorata, G. Redaelli, and A. Vulpiani, Physica D. 159, 58 (2001).

[13] P. H. Haynes, D. A. Poet, and E. F. Shuckburgh, J. Atmos. Sci. 64, 3640 (2007).

[14] J. Pedlosky, Geophysical fluid dynamics (New-York: Springer-Verlag, 1987).

[15] H.L. Kuo, J. Meteorol. 6, 105 (1949).

[16] S. Shinohara and Y. Aizawa, Progr. Theor. Phys. 100, 219 (1998).

[17] M. V. Budyansky, M. Yu. Uleysky, and S. V. Prants, Phys. Rev. E 79, 056215 (2009).

[18] M. Yu. Uleysky, M. V. Budyansky, and S. V. Prants, http://dynalab.poi.dvo.ru/papers/mult1.avi and http://dynalab.poi.dvo.ru/papers/mult2.avi 